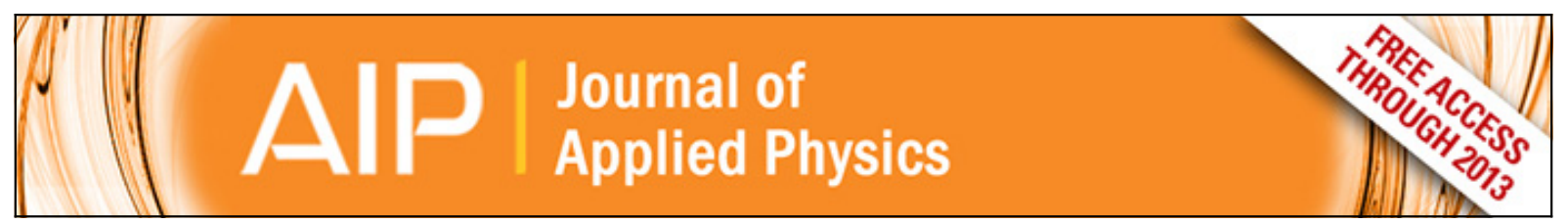

X-ray diffraction study on pressure-induced phase transformations and the equation of state of ZnGa2Te4

D. Errandonea, R. S. Kumar, O. Gomis, F. J. Manjón, V. V. Ursaki, and I. M. Tiginyanu

Citation: Journal of Applied Physics 114, 233507 (2013); doi: 10.1063/1.4851735

View online: http://dx.doi.org/10.1063/1.4851735

View Table of Contents: http://scitation.aip.org/content/aip/journal/jap/114/23?ver=pdfcov

Published by the AIP Publishing

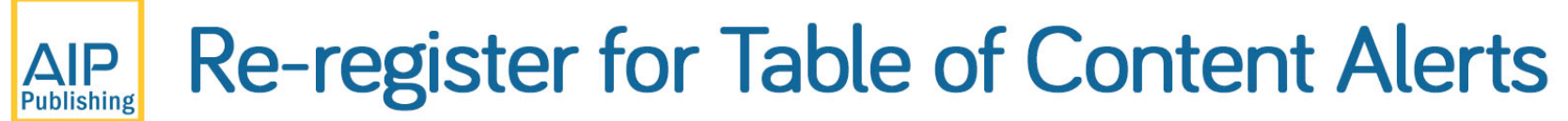

Create a profile. 


\title{
X-ray diffraction study on pressure-induced phase transformations and the equation of state of $\mathrm{ZnGa}_{2} \mathrm{Te}_{4}$
}

\author{
D. Errandonea, ${ }^{1, a)}$ R. S. Kumar, ${ }^{2}$ O. Gomis, ${ }^{3}$ F. J. Manjón, ${ }^{4}$ V. V. Ursaki, ${ }^{5}$ \\ and I. M. Tiginyanu ${ }^{5}$ \\ ${ }^{1}$ Departamento de Física Aplicada-ICMUV, MALTA Consolider Team, Universidad de Valencia, \\ Edificio de Investigación, C/Dr. Moliner 50, Burjassot, 46100 Valencia, Spain \\ ${ }^{2}$ High Pressure Science and Engineering Center, Department of Physics and Astronomy, \\ University of Nevada Las Vegas, 4505 Maryland Parkway, Las Vegas, Nevada 89154-4002, USA \\ ${ }^{3}$ Centro de Tecnologías Físicas: Acústica, Materiales y Astrofísica, MALTA Consolider Team, \\ Universitat Politècnica de València, 46022 València, Spain \\ ${ }^{4}$ Instituto de Diseño para la Fabricación y Producción Automatizada, MALTA Consolider Team, \\ Universitat Politècnica de València, 46022 València, Spain \\ ${ }^{5}$ Institute of Applied Physics, Academy of Sciences of Moldova, 2028 Chisinau, Moldova
}

(Received 22 October 2013; accepted 5 December 2013; published online 18 December 2013)

\begin{abstract}
We report on high-pressure $\mathrm{x}$-ray diffraction measurements up to $19.8 \mathrm{GPa}$ in zinc digallium telluride $\left(\mathrm{ZnGa}_{2} \mathrm{Te}_{4}\right)$ at room temperature. An irreversible structural phase transition takes place at pressures above $12.1 \mathrm{GPa}$ and upon decompression a third polymorph of $\mathrm{ZnGa}_{2} \mathrm{Te}_{4}$ was recovered as a metastable phase at pressures below 2.9 GPa. Rietveld refinements were carried out for the three detected polymorphs, being their possible crystal structures reported. The axial compressibilities for the low-pressure phase of $\mathrm{ZnGa}_{2} \mathrm{Te}_{4}$ have been determined as well as the equation of state of the low- and high-pressure phases. The reported results are compared with those available in the literature for related compounds. Pressure-induced coordination changes and transition mechanisms are also discussed. (C) 2013 AIP Publishing LLC. [http://dx.doi.org/10.1063/1.4851735]
\end{abstract}

\section{INTRODUCTION}

Defect-chalcopyrite (DC) and defect-stannite (DS) compounds are members of the adamantine-type $\mathrm{AB}_{2} \mathrm{X}_{4}$ family of semiconductors. These materials are currently being investigated due to their diversity of applications which include optoelectronics, non-linear optics, and electro-optics among others. ${ }^{1}$ Zinc digallium telluride $\left(\mathrm{ZnGa}_{2} \mathrm{Te}_{4}\right)$ is part of this family of compounds. It has been proposed as a promising material for developing phase-change memory devices. $^{2}$ To develop these potential applications, a correct determination of the electronic properties of $\mathrm{ZnGa}_{2} \mathrm{Te}_{4}$ is needed, for which a precise knowledge of its crystal structure is fundamental. This structure has been studied by x-ray diffraction $(\mathrm{XRD})^{3}$ and $a b$ initio calculations. ${ }^{4}$ According to previous experiments, $\mathrm{ZnGa}_{2} \mathrm{Te}_{4}$ crystallizes in the tetragonal DC structure (space group $I \overline{4}$ ), however, its structure has not been fully refined and its yet under debate being also the DS structure a good candidate for it (space group $I \overline{4} 2 m){ }^{5}$

High-pressure (HP) structural studies on $\mathrm{AB}_{2} \mathrm{X}_{4}$ compounds have received increasing attention in the last years. Among other techniques, they have been studied by XRD, being several pressure-induced transitions reported. ${ }^{6-15}$ The reversibility of these transitions is subject of dispute. In some cases, it has been found that the transitions are not reversible, being metastable polymorphs recovered at ambient conditions after decompression. These polymorphs have a smaller electronic band gap than the ambient-pressure stable polymorph, ${ }^{16}$ opening the door to novel applications for

\footnotetext{
a) Author to whom correspondence should be addressed. Electronic mail: daniel.errandonea@uv.es
}

$\mathrm{AB}_{2} \mathrm{X}_{4}$ semiconductors. In contrast with other $\mathrm{AB}_{2} \mathrm{X}_{4}$ compounds, only few works have been devoted to the study of the structural properties of $\mathrm{ZnGa}_{2} \mathrm{Te}_{4}{ }^{3,4}$ and no records of HP studies can be found in the literature. Here, we report room-temperature (RT) synchrotron HP XRD measurements up to $19.8 \mathrm{GPa}$ to study into detail the structural properties of the low-pressure phase of $\mathrm{ZnGa}_{2} \mathrm{Te}_{4}$ and the possible occurrence of structural phase transitions. The RT equation of state (EOS) of the different polymorphs found in $\mathrm{ZnGa}_{2} \mathrm{Te}_{4}$ will be presented. Technical aspects of the experiments are described in Sec. II. Results are presented and discussed in Sec. III and conclusions are summarized in Sec. IV.

\section{EXPERIMENTAL DETAILS}

Single crystals of $\mathrm{ZnGa}_{2} \mathrm{Te}_{4}$ were grown by chemical vapor method using iodine as a transport agent. ${ }^{17}$ The as-grown crystals of uniform dark-red color represent triangular prisms with mirror-like surfaces. Their chemical composition was verified by energy-dispersive $\mathrm{x}$-ray analysis with a Phillips XL-30 scanning electron microscope. No impurities have been detected and the determined stoichiometry of the crystals corresponds to pure $\mathrm{ZnGa}_{2} \mathrm{Te}_{4}$ with a precision of $0.2 \%$. Angle-dispersive XRD experiments were carried out at RT under pressure up to $19.8 \mathrm{GPa}$ using a diamond-anvil cell (DAC) at Sector 16-IDB of the HPCAT, at the Advanced Photon Source (APS). Measurements were also carried out on decompression. Experiments were performed with an incident monochromatic wavelength $(0.3681 \AA)$. The sample used was a $10-\mu$ m thick pre-pressed pellet prepared using a finely ground powder obtained from the as grown single crystals. The pellet was loaded in a 
$130-\mu \mathrm{m}$ hole of a $50-\mu \mathrm{m}$ thick rhenium gasket in a DAC with diamond-culet sizes of $350 \mu \mathrm{m}$. Ruby grains were loaded with the sample for pressure calibration ${ }^{18}$ and a $4: 1$ methanol-ethanol mixture was used as pressure-transmitting medium. ${ }^{19,20}$ Pressure was assumed as the average of the pressure determined from different rubies and the accuracy assumed to be the standard deviation from average. In order to ensure the accuracy of the experiments, special attention was paid to avoiding the sample or the ruby grains bridging with the anvils. The $\mathrm{x}$-ray beam was focused down to $10 \times 10 \mu \mathrm{m}^{2}$ using Kirkpatrick-Baez mirrors. The images were collected using a MAR345 image plate located $380 \mathrm{~mm}$ away from the sample. The collected images were integrated and corrected for distortions using FIT2D. ${ }^{21}$ The structure refinements were performed using the POWDERCELL ${ }^{22}$ program package.

\section{RESULTS AND DISCUSSION}

\section{A. Crystal structure at ambient conditions}

Figure 1 shows an XRD pattern collected in $\mathrm{ZnGa}_{2} \mathrm{Te}_{4}$ at ambient pressure. It could be satisfactorily indexed with both the DC $(I \overline{4})$ or the DS $(I \overline{4} 2 m)$ structures. Rietveld refinements considering both structures were carried out.

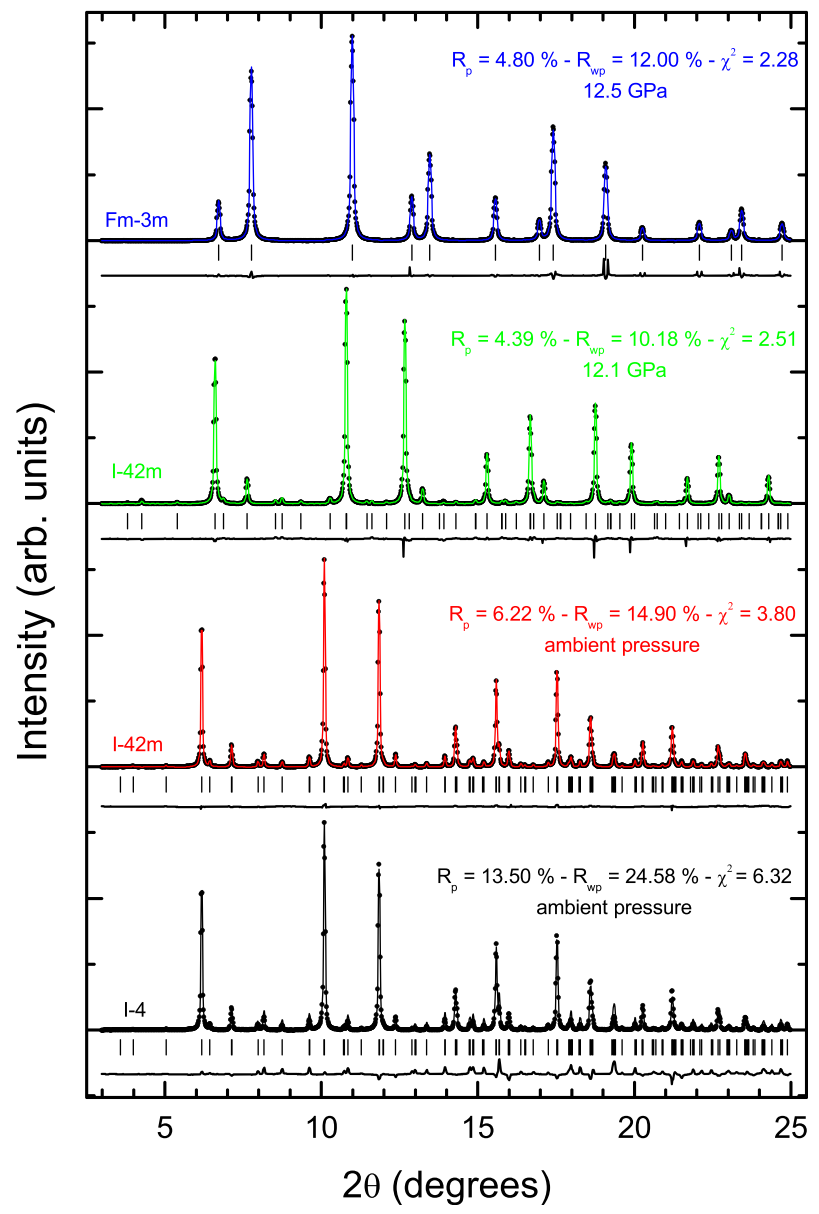

FIG. 1. RT XRD patterns of $\mathrm{ZnGa}_{2} \mathrm{Te}_{4}$ at selected pressures on upstroke. In all diagrams, the background was subtracted. The Rietveld refinements are also shown. Dots: experiments. Solid lines: calculated diffraction profiles and residuals. Calculated Bragg reflections are indicated with vertical ticks. $\mathrm{R}$-factors and the $\chi^{2}$ obtained from the refinements are also given.
The results of the Rietveld refinement obtained for both structures are shown in Fig. 1. In addition to the unit-cell parameters, we also refined the Te positions. The positions of $\mathrm{Zn}$ and Ga are fixed by the structural symmetry of both structures. Since the site occupancy factor (SOF) and the atomic displacement factors are correlated and more sensitive to background subtraction than positional parameters, the SOFs were constrained to the values given in Table I and the overall displacement factor, $B$, was fixed to $0.5 \AA^{2} .{ }^{23}$ In this way, we reduced the number of free parameters used in the refinement. In the Rietveld analysis, we assumed as initial values for the Te coordinates those of Se in DS- $\mathrm{ZnGa}_{2} \mathrm{Se}_{4}$ (Ref. 9) and of $\mathrm{S}$ in DC-CdGa $2 \mathrm{~S}_{4}$ (Ref. 9). Table I provides the structural parameters obtained from the Rietveld refinements. As can be seen in Fig. 1, the results are more consistent with the DS structure on the basis of the refinement residuals. The parameters characterizing the goodness of fit (R-values and $\left.\chi^{2}\right)^{24}$ also support the assignment of the DS structure to the ambient conditions crystal structure of $\mathrm{ZnGa}_{2} \mathrm{Te}_{4}$. The refined structure agrees with the structure proposed by Hahn et al. ${ }^{5}$ who also assigned the space group $I \overline{4} 2 m$ to the structure of $\mathrm{ZnGa}_{2} \mathrm{Te}_{4}$. In contrast, our results contradict the conclusions of Rashmi and Dhawan ${ }^{3}$ who assigned the space group $I \overline{4}$ to the same structure. On the other hand, our unit-cell parameters $a=5.922(1) \AA$ and $c=11.809(2) \AA$ are very similar to those previously reported. ${ }^{3,5}$ The present results also correlate quite well with the structure calculated for DS $\mathrm{ZnGa}_{2} \mathrm{Te}_{4}$ using density-functional theory. ${ }^{4}$ According with the refined structure, $\mathrm{Ga}$ atoms at the Wyckoff position 2a are four-coordinated by Te atoms, the bond distance being 2.627(4) $\AA$. On the other hand, Ga and $\mathrm{Zn}$ atoms at the Wyckoff position $4 \mathrm{~d}$ are four-coordinated by Te atoms, the bond distance being 2.617(4) A.

\section{B. Pressure-induced phase transitions}

Upon compression, we did not observe any substantial change in the XRD patterns up to P $>12.1 \mathrm{GPa}$. In Fig. 1, it can be seen that the diffraction pattern can be still assigned to the tetragonal DS structure $(I \overline{4} 2 \mathrm{~m})$ at $12.1 \mathrm{GPa}$. The goodness of fit of the structural refinement with this model is similar at $12.1 \mathrm{GPa}$ to that obtained at ambient pressure with the same model (see Fig. 1). Table II gives the structural parameters obtained from the Rietveld refinement at $12.1 \mathrm{GPa}$. By comparing Tables I and II, it can be seen that at $12.1 \mathrm{GPa}$ the

TABLE I. Atomic positions and SOF of $\mathrm{ZnGa}_{2} \mathrm{Te}_{4}$ at ambient pressure.

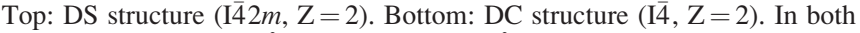
structures $a=5.922(1) \AA$ and $c=11.809(2) \AA$.

\begin{tabular}{lccccc}
\hline \hline Atom & Site & $\mathrm{x}$ & $\mathrm{y}$ & $\mathrm{z}$ & $\mathrm{SOF}$ \\
\hline $\mathrm{Ga}$ & $2 \mathrm{a}$ & 0 & 0 & 0 & 1 \\
$\mathrm{Ga}$ & $4 \mathrm{~d}$ & 0 & $1 / 2$ & $1 / 4$ & 0.5 \\
$\mathrm{Zn}$ & $4 \mathrm{~d}$ & 0 & $1 / 2$ & $1 / 4$ & 0.5 \\
$\mathrm{Te}$ & $8 \mathrm{i}$ & $0.2664(9)$ & $0.2664(9)$ & $0.1175(8)$ & 1 \\
$\mathrm{Ga}$ & $2 \mathrm{~b}$ & 0 & 0 & $1 / 2$ & 1 \\
$\mathrm{Ga}$ & $2 \mathrm{c}$ & 0 & $1 / 2$ & $1 / 4$ & 1 \\
$\mathrm{Zn}$ & $2 \mathrm{a}$ & 0 & 0 & 0 & 1 \\
$\mathrm{Te}$ & $8 \mathrm{~g}$ & $0.2752(8)$ & $0.2340(9)$ & $0.1357(8)$ & 1 \\
\hline \hline
\end{tabular}


TABLE II. Top: Atomic positions and SOF of DS-ZnGa $\mathrm{Te}_{4}$ at $12.1 \mathrm{GPa}$ $(I \overline{4} 2 m, \mathrm{Z}=2)$. Bottom: same for DR-ZnGa $\mathrm{Te}_{4}$ at $12.5 \mathrm{GPa}(F m \overline{3} m, \mathrm{Z}=1)$. In the DS structure $a=5.532(1) \AA$ and $c=11.064(2) \AA$. In the DR structure $a=5.432(2) \AA$

\begin{tabular}{lccccc}
\hline \hline Atom & Site & $\mathrm{x}$ & $\mathrm{y}$ & $\mathrm{z}$ & $\mathrm{SOF}$ \\
\hline $\mathrm{Ga}$ & $2 \mathrm{a}$ & 0 & 0 & 0 & 1 \\
$\mathrm{Ga}$ & $4 \mathrm{~d}$ & 0 & $1 / 2$ & $1 / 4$ & 0.5 \\
$\mathrm{Zn}$ & $4 \mathrm{~d}$ & 0 & $1 / 2$ & $1 / 4$ & 0.5 \\
$\mathrm{Te}$ & $8 \mathrm{i}$ & $0.2539(9)$ & $0.2539(9)$ & $0.1219(8)$ & 1 \\
$\mathrm{Ga}$ & $4 \mathrm{a}$ & 0 & 0 & 0 & 0.5 \\
$\mathrm{Zn}$ & $4 \mathrm{a}$ & 0 & 0 & 0 & 0.25 \\
$\mathrm{Te}$ & $4 \mathrm{~b}$ & $1 / 2$ & $1 / 2$ & $1 / 2$ & 1 \\
\hline \hline
\end{tabular}

ratio $c / a$ is equal to 2 and the $\mathrm{x}$ and $\mathrm{y}$ coordinates of Te approaches the value 0.25 and the $\mathrm{z}$ coordinate approaches the value 0.125 . Thus, pressure induces a symmetry increase of the crystal structure of $\mathrm{ZnGa}_{2} \mathrm{Te}_{4}$ so that it resembles a pseudo-rocksalt structure at $12.1 \mathrm{GPa}$ with the $c$-axis doubled. At this pressure, the DS structure has Te atoms arranged in a similar fashion as in an fcc lattice and, similarly, $\mathrm{Zn}$ and $\mathrm{Ga}$ atoms also form an fcc lattice with one quarter of the atomic sites empty. The parameter of the cell of each of these fcc substructures of the DS phase is around $5.532 \AA$, being the distances between Te atoms and cations at $2 \mathrm{a}$ and $4 \mathrm{~d}$ sites $2.402(5) \AA$ and $2.415(5) \AA$, respectively. All the facts described above about the pressure-induced symmetry increase of $\mathrm{ZnGa}_{2} \mathrm{Te}_{4}$ have been also observed in $\mathrm{ZnGa}_{2} \mathrm{Se}_{4}{ }^{9}$, suggesting that they can be a common feature of DS compounds. In particular, in both compounds the displacement of the anions towards the position $(0.25,0.25$, 0.125 ) and the increase of the axial ratio to reach $c / a=2$ make the coordination tetrahedra in both compounds more regular. In special, the tetrahedral bond angles under compression approach all the ideal value of $109.5^{\circ}$. A meaningful explanation of the observed symmetry increase is beyond the scope of this work. However, a possible cause for it could be the fact that in $\mathrm{ZnGa}_{2} \mathrm{Te}_{4}$ and $\mathrm{ZnGa}_{2} \mathrm{Se}_{4}$ at ambient pressure the distance between anions is shorter along $c$ than in the perpendicular axes. Thus, the opposite behavior is expected for the electrostatic repulsion among anions, making therefore the $c$-axis less compressible than the $a$-axis as found.

Upon further compression, at $12.3 \mathrm{GPa}$, we found the appearance of extra diffraction peaks, which indicates the onset of a structural phase transition. At $12.5 \mathrm{GPa}$, the new peaks fully develop and the peaks of the low-pressure phase cannot be detected anymore. As found in $\mathrm{ZnGa}_{2} \mathrm{Se}_{4}$ and related compounds, ${ }^{8-13}$ we found that the XRD patterns of the HP structure of $\mathrm{ZnGa}_{2} \mathrm{Te}_{4}$ can be assigned to a cubic rocksalt-type structure $(F m \overline{3} m)$, where the Te atoms are located at the $4 \mathrm{~b}$ site, while the $\mathrm{Zn}$ and $\mathrm{Ga}$ atoms are located at the $4 \mathrm{a}$ sites with a partial SOF. Results of the Rietveld refinements indicating the good match of this disordered rocksalt-type (DR) structure with the experimental results are shown in Fig. 1. Details of the structural model are given in Table II. We would like to stress here, that in spite the DR structure was previously assigned to the HP phase of other members of the $\mathrm{AB}_{2} \mathrm{X}_{4}$ family, ${ }^{8-13}$ this is the first time that it is assigned using enough Bragg peaks (fourteen) to be confident on the structural assignment. This was possible thanks to the large unit-cell volume of $\mathrm{ZnGa}_{2} \mathrm{Te}_{4}$ and the large angular aperture of the DAC used in the experiments $\left(2 \theta<25^{\circ}\right)$. The occurrence of the DS to DR transition, observed at $\mathrm{P}>12.1 \mathrm{GPa}$, implies the completion of the symmetry increase that occurs in the low-pressure phase upon compression. Note that the unit-cell parameter of the cubic structure, $a=5.432(2) \AA$, at $12.5 \mathrm{GPa}$ implies an $1.7 \%$ contraction from the parameters defining the pseudo-rocksalt framework that can be drawn in the low-pressure tetragonal phase at $12.1 \mathrm{GPa}$. We have estimated a relative volume change per formula unit of $-4.8 \%$ at $12.5 \mathrm{GPa}$, thus indicating that the DS to DR phase transition is a first-order transition. On the other hand, a comparison of the fcc sublattices of cations and anions in both DS and DR structures around $12 \mathrm{GPa}$ (see Fig. 2) shows that a relative shift of the two fcc sublattices of the DS structure is needed (along the [111] direction or an equivalent one) in order that atoms of each fcc sublattice locate at the octahedral holes of the other

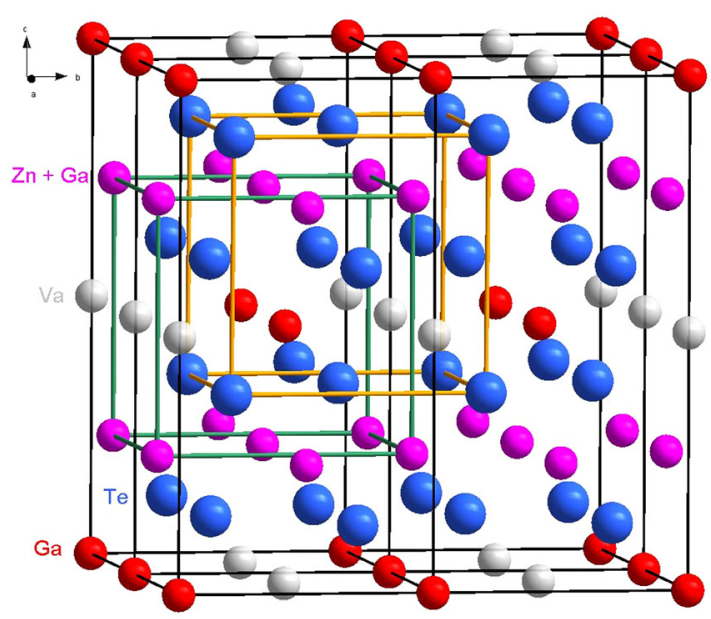

(a)

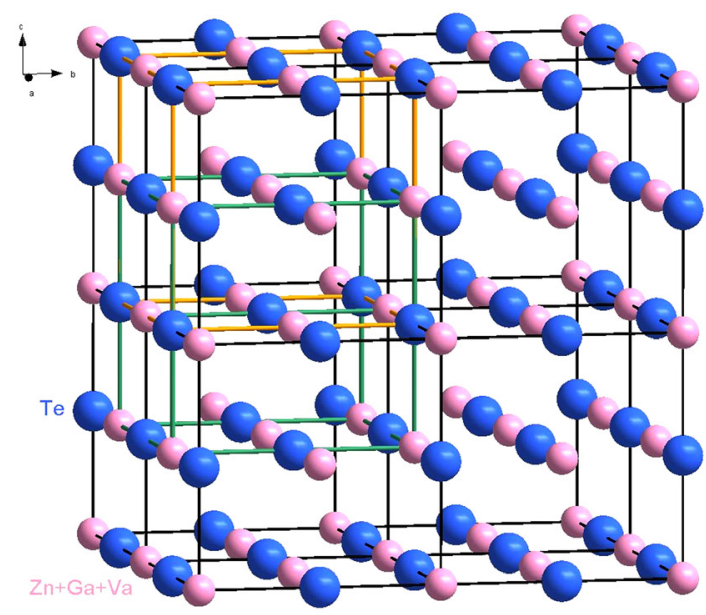

(b)

FIG. 2. Structure of DS-ZnGa $\mathrm{Te}_{4}$ at $12.1 \mathrm{GPa}$ (a) and of $\mathrm{DR}-\mathrm{ZnGa}_{2} \mathrm{Te}_{4}$ at 12.5 $\mathrm{GPa}$ (b). For the sake of comparison, the cubic unit cells of the fcc-like sublattices of cations and anions in both structures are depicted in green and orange, respectively. Different atoms are identified in the figure. 
sublattice, thus transforming the DS into the DR structure. The relative shift of the two fcc sublattices corresponds to the translation of Te atoms from tetrahedral voids to octahedral voids in the cation fcc sublattice. This fact suggests that the DS to DR transition might be a displacive transformation. Additionally, the described transition comprises a change in the coordination of both cations and anions from tetrahedral to octahedral, whose consequence is an increase of the cation-Te distance to 2.719 (4) $\AA$ at $12.5 \mathrm{GPa}$ in the DR phase compared to the that in the DS phase at $12.1 \mathrm{GPa}$ whose average is $2.411 \AA$.

The DR phase remains stable up to the highest pressure reached in the experiments (19.8 GPa) as it is evidenced by the Rietveld refinement of the XRD pattern at this pressure to the DR phase shown at the bottom of Fig. 3 (residuals are small, and the R-factors and $\chi^{2}$ indicate a good fit). Upon decompression, the DR structure is retained down to $2.9 \mathrm{GPa}$. In Fig. 3, we show how the XRD pattern measured at this pressure can be satisfactorily fitted with the same DR structure. However, after a subsequent decompression step in which we reached ambient pressure we observed an XRD pattern that cannot be explained either by the DS or the DR structures of $\mathrm{ZnGa}_{2} \mathrm{Te}_{4}$ (see Fig. 3). The irreversibility of the phase transition was already observed in other related $\mathrm{AB}_{2} \mathrm{X}_{4}$ compounds ${ }^{8,10,11,13,25}$ and it was proposed that the recovered samples transformed to a metastable disordered zincblende-type (DZ) structure $(F \overline{4} 3 m)$ at ambient

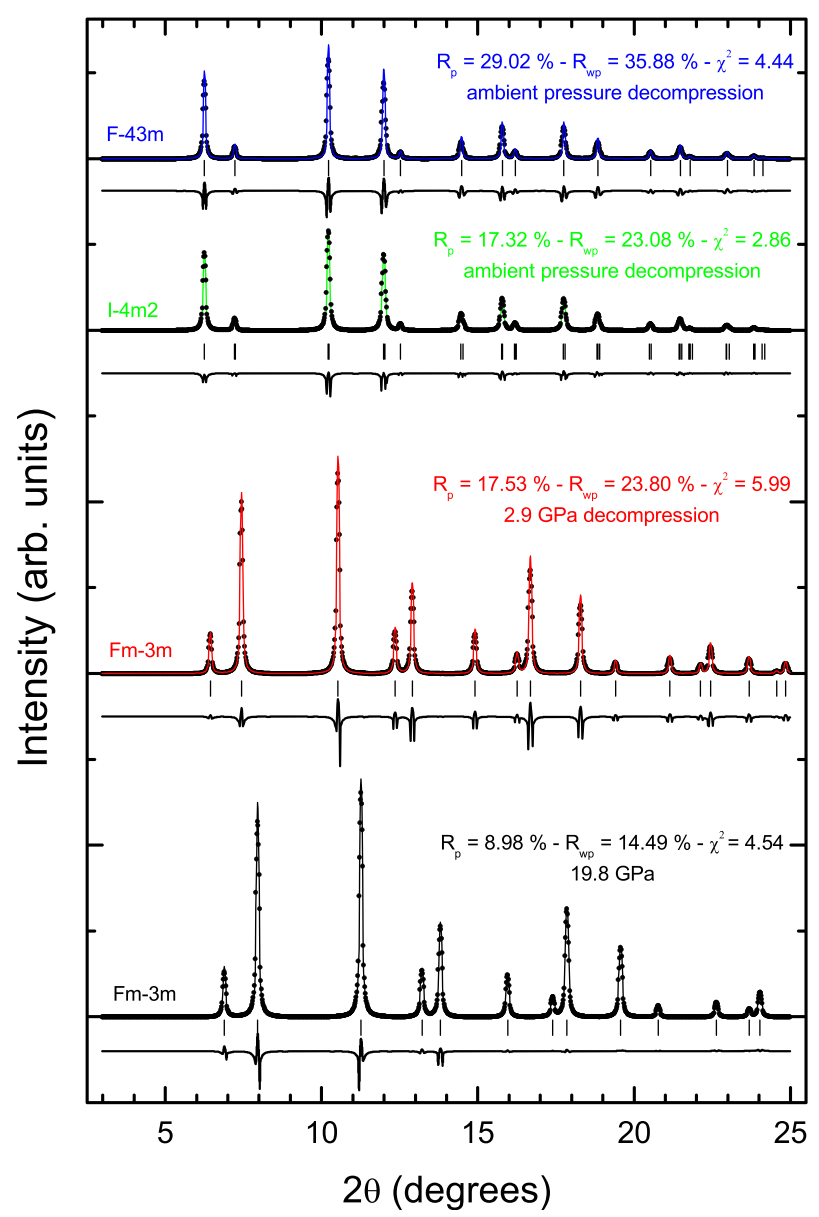

FIG. 3. Same as Fig. 1 but showing XRD patterns collected on downstroke. pressure. ${ }^{8,10,11,13}$ In our case, we have been able to successfully reproduce the diffraction patterns collected at ambient pressure after decompression by assuming the DZ structure. Residuals and parameters indicating the quality of the structural refinement are given in Fig. 3 while the parameters of the refined structure are given in Table III. We would also like to mention here that during the structural refinement of the pattern at ambient pressure we obtained a decrease of the residuals of the structural refinement and consequently a better fit by reducing the symmetry of the DZ structure to the tetragonal subgroup $(I \overline{4} m 2)$ of the $F \overline{4} 3 m$ structure (see Fig. 3). Details of this tetragonal structure are given in Table III. Note that this structure reduces to the zincblende structure if $c / a=\sqrt{2}$, which is close to the axial ratio we obtain for the tetragonal structure (1.407). The small variation of $c / a$ from $\sqrt{2}$ cause a subtle splitting of many Bragg peaks and therefore the tetragonal structure accounts better for the width of the diffraction peaks. However, the width of the peaks can be also explained by other factors like presence of defects in the sample, micro-strains and grain size. ${ }^{26}$ Therefore, on the basis of the reported results we can only state that decompression of $\mathrm{ZnGa}_{2} \mathrm{Te}_{4}$ from the DR structure results in a metastable phase at ambient pressure whose structure is related to the DZ structure previously proposed for related compounds. ${ }^{8,10,11,13,25}$ The crystal structure cannot be fully solved from HP powder XRD experiments and the accurate solution of the crystal structure of the recovered sample may probably need the performance of HP single-crystal experiments. ${ }^{27}$

\section{Room-temperature equation of state of the DS and DR phases}

The pressure evolution of the unit-cell parameters of the low-(DS) and high-pressure (DR) phases has been obtained from the structural refinements. Results are listed in Tables IV and V. Figure 4 shows the pressure dependence of $a$ and $c$ for $\mathrm{DS}-\mathrm{ZnGa}_{2} \mathrm{Te}_{4}$. The axial compressibilities at zero pressure $\left(\kappa_{x}=-\frac{\partial \ln x}{\partial P}\right)$ obtained by fitting of a Murnaghan $\operatorname{EOS}^{28}$ are $\kappa_{\mathrm{a}}=8.48(6) \times 10^{-3} \mathrm{GPa}^{-1}, \kappa_{\mathrm{c}}=8.09(7) \times 10^{-3}$ $\mathrm{GPa}^{-1}$; i.e., the $c$-axis is $\sim 5 \%$ less compressible than the $a$-axis. This fact is illustrated in the inset of Fig. 4 where we show the pressure dependence of the $c / a$ ratio. As commented above, this ratio gradually increases from 1.994 at ambient pressure to 2 at $12.1 \mathrm{GPa}$.

TABLE III. Top: Atomic positions and SOF of DZ-ZnGa $\mathrm{Te}_{4}$ at ambient pressure $(F \overline{4} 3 m, Z=1)$. Bottom: same for proposed metastable tetragonal structure of $\mathrm{ZnGa}_{2} \mathrm{Te}_{4}$ at ambient pressure $(I \overline{4} m 2, Z=1 / 2)$. In the DZ structure $a=5.843(6) \AA$. In the tetragonal structure $a=4.139(5) \AA$ and $c=5.823(5) \AA$.

\begin{tabular}{lccccc}
\hline \hline Atom & Site & $\mathrm{x}$ & $\mathrm{y}$ & $\mathrm{z}$ & $\mathrm{SOF}$ \\
\hline $\mathrm{Ga}$ & $4 \mathrm{a}$ & 0 & 0 & 0 & 0.5 \\
$\mathrm{Zn}$ & $4 \mathrm{a}$ & 0 & 0 & 0 & 0.25 \\
$\mathrm{Te}$ & $4 \mathrm{c}$ & $1 / 4$ & $1 / 4$ & $1 / 4$ & 1 \\
$\mathrm{Ga}$ & $2 \mathrm{a}$ & 0 & 0 & 0 & 0.5 \\
$\mathrm{Zn}$ & $2 \mathrm{a}$ & 0 & 0 & 0 & 0.25 \\
$\mathrm{Te}$ & $2 \mathrm{c}$ & 0 & $1 / 2$ & $1 / 4$ & 1 \\
\hline
\end{tabular}


TABLE IV. Experimental pressure (P), unit-cell parameters $(a, c)$ and volume (V) of DS- $\mathrm{ZnGa}_{2} \mathrm{Te}_{4}$ with their standard deviations (esd).

\begin{tabular}{lccccccc}
\hline \hline$P$ & esd $(P)$ & $a$ & esd $(a)$ & $c$ & esd $(c)$ & $V$ & esd $(V)$ \\
GPa & GPa & $\AA$ & $\AA$ & $\AA$ & $\AA$ & $\AA^{3}$ & $\AA^{3}$ \\
\hline 0.00 & 0.02 & 5.922 & 0.001 & 11.809 & 0.002 & 414.14 & 0.21 \\
0.10 & 0.02 & 5.917 & 0.001 & 11.799 & 0.002 & 413.09 & 0.21 \\
0.30 & 0.02 & 5.909 & 0.001 & 11.784 & 0.002 & 411.45 & 0.21 \\
0.50 & 0.02 & 5.899 & 0.001 & 11.764 & 0.002 & 409.37 & 0.21 \\
0.80 & 0.02 & 5.885 & 0.001 & 11.738 & 0.002 & 406.52 & 0.21 \\
1.10 & 0.02 & 5.869 & 0.001 & 11.707 & 0.002 & 403.25 & 0.21 \\
1.50 & 0.02 & 5.853 & 0.001 & 11.677 & 0.002 & 400.03 & 0.21 \\
2.00 & 0.02 & 5.830 & 0.001 & 11.632 & 0.002 & 395.36 & 0.20 \\
2.80 & 0.02 & 5.801 & 0.001 & 11.577 & 0.002 & 389.58 & 0.20 \\
3.60 & 0.02 & 5.769 & 0.001 & 11.516 & 0.002 & 383.27 & 0.20 \\
4.30 & 0.02 & 5.745 & 0.001 & 11.470 & 0.002 & 378.57 & 0.20 \\
5.30 & 0.02 & 5.711 & 0.001 & 11.407 & 0.002 & 372.05 & 0.20 \\
6.00 & 0.02 & 5.688 & 0.001 & 11.363 & 0.002 & 367.63 & 0.19 \\
7.00 & 0.02 & 5.659 & 0.001 & 11.308 & 0.002 & 362.13 & 0.19 \\
7.80 & 0.02 & 5.638 & 0.001 & 11.270 & 0.002 & 358.24 & 0.19 \\
8.70 & 0.02 & 5.612 & 0.001 & 11.219 & 0.002 & 353.34 & 0.19 \\
9.70 & 0.02 & 5.586 & 0.001 & 11.171 & 0.002 & 348.57 & 0.19 \\
10.60 & 0.04 & 5.566 & 0.001 & 11.131 & 0.002 & 344.84 & 0.19 \\
11.50 & 0.04 & 5.545 & 0.001 & 11.089 & 0.002 & 340.95 & 0.18 \\
12.10 & 0.04 & 5.532 & 0.001 & 11.064 & 0.002 & 338.59 & 0.18 \\
\hline \hline
\end{tabular}

Figure 5 shows the pressure dependence of the volume for the DS and DR phases. EOS parameters for both phases were determined by least-squares fits of 2nd (BM2) and 3rd (BM3) order Birch-Murnaghan $\mathrm{EOSs}^{29}$ to the pressurevolume $(\mathrm{P}-\mathrm{V})$ data. Weights derived from the experimental uncertainties, in both pressure and volume, were assigned to each data point in the fits. They were carried out with the EoSFit software (v5.2). ${ }^{30}$ Table VI summarizes the zeropressure values of the volume $\left(\mathrm{V}_{0}\right)$, bulk modulus $\left(\mathrm{B}_{0}\right)$, and its pressure derivative $\left(\mathrm{B}_{0}{ }^{\prime}\right)$ obtained from the fits of both DS and DR phases. The obtained values for the weighted chi-squared, $\chi^{2}{ }_{\mathrm{w}}$, are also given in the table. For both DS and DR phases, we found that the fit does not improve by leaving $\mathrm{B}_{0}{ }^{\prime}$ as a free parameter. This result indicates that a 3rd order expansion of the EOS is not required to fit our experimental

TABLE V. Experimental pressure (P), unit-cell parameters (a) and volume (V) of DR- $\mathrm{ZnGa}_{2} \mathrm{Te}_{4}$ with their standard deviations (esd). Last column indicates whether the measurement was done on compression or decompression.

\begin{tabular}{lcccccc}
\hline \hline $\begin{array}{l}P \\
\mathrm{GPa}\end{array}$ & $\begin{array}{c}\mathrm{esd}(P) \\
\mathrm{GPa}\end{array}$ & $\begin{array}{c}a \\
\AA\end{array}$ & $\begin{array}{c}\mathrm{esd}(a) \\
\AA\end{array}$ & $\begin{array}{c}V \\
\AA^{3}\end{array}$ & $\begin{array}{c}\text { esd }(V) \\
\AA^{3}\end{array}$ & Measurement \\
\hline 12.50 & 0.04 & 5.432 & 0.002 & 160.28 & 0.18 & Upstroke \\
14.70 & 0.04 & 5.391 & 0.002 & 156.68 & 0.17 & Upstroke \\
16.90 & 0.04 & 5.351 & 0.002 & 153.22 & 0.17 & Upstroke \\
18.10 & 0.04 & 5.333 & 0.002 & 151.68 & 0.17 & Upstroke \\
19.80 & 0.04 & 5.305 & 0.002 & 149.30 & 0.17 & Upstroke \\
13.2 & 0.2 & 5.419 & 0.002 & 159.13 & 0.18 & Downstroke \\
11.5 & 0.2 & 5.458 & 0.002 & 162.59 & 0.18 & Downstroke \\
9.3 & 0.2 & 5.501 & 0.002 & 166.47 & 0.18 & Downstroke \\
5.6 & 0.2 & 5.574 & 0.002 & 173.18 & 0.19 & Downstroke \\
2.9 & 0.2 & 5.674 & 0.002 & 182.67 & 0.19 & Downstroke \\
\hline
\end{tabular}

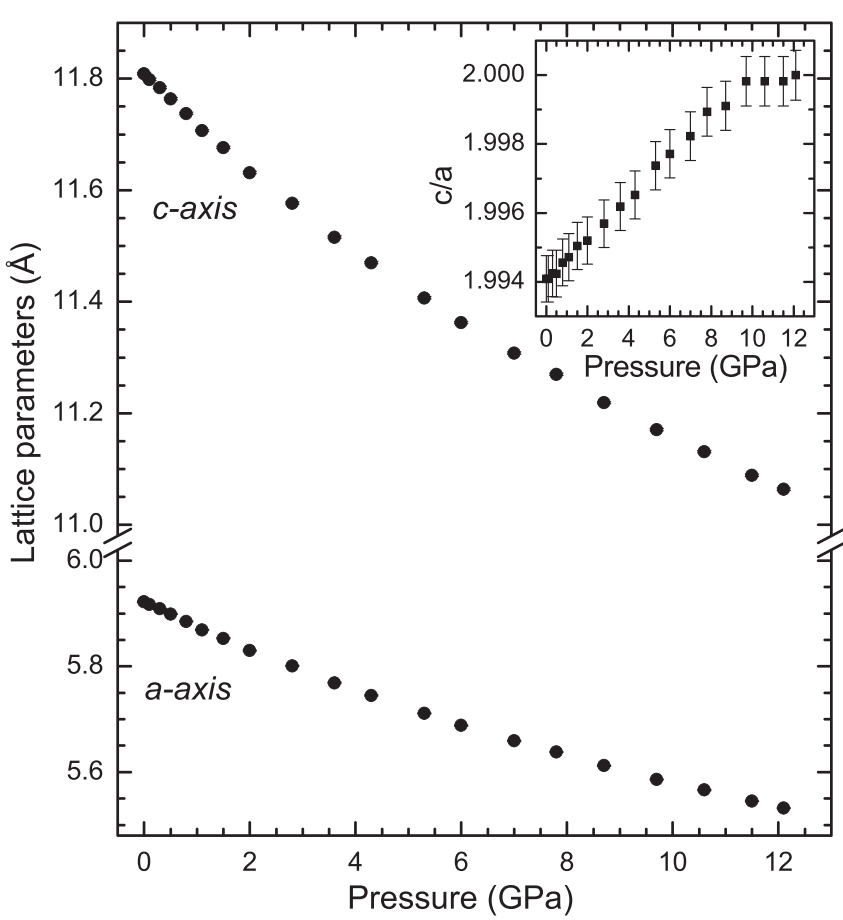

FIG. 4. Pressure evolution of unit-cell parameters in $\mathrm{DS}-\mathrm{ZnGa}_{2} \mathrm{Te}_{4}$. The inset shows the pressure evolution of the $c / a$ ratio. Where not shown, error bars are smaller than symbols size.

data. In Fig. 5, it can be seen that both BM2 and BM3 fits accurately reproduce the experimental results. According to our results DS- $\mathrm{ZnGa}_{2} \mathrm{Te}_{4}$ is one of the compounds of the adamantine-type $\mathrm{AB}_{2} \mathrm{X}_{4}$ family with the smallest bulk modulus. ${ }^{15}$ The bulk modulus of DR- $\mathrm{ZnGa}_{2} \mathrm{Te}_{4}$ is $\sim 20 \%$ larger than that of the low-pressure phase, which is consistent with the volume collapse observed at the transition (see Fig. 5). In particular, the DR phase has a volume $4.8 \%$ smaller than the DS phase at $12.5 \mathrm{GPa}$ as previously commented. In addition, if we compare the normalized volumes of the DS, DR, and

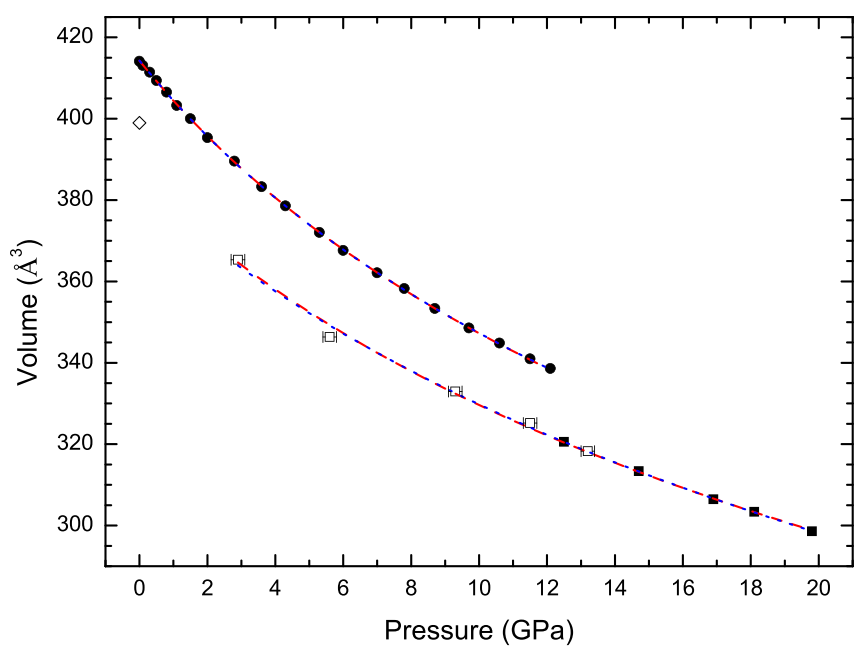

FIG. 5. Pressure evolution of unit-cell volume for different phases of $\mathrm{ZnGa}_{2} \mathrm{Te}_{4}$ : Circles (DS), squares (DR), and diamond (DZ-like). To facilitate comparison for the three phases we have plotted the double of the unit-cell volume for DR and DZ phases. Solid (empty) symbols represent data measured on compression (decompression). Where not shown error bars are smaller than symbol size. The red dashed (blue dotted) lines represent 2 nd (3rd) order BM EOSs. 
TABLE VI. EOS parameters and $\chi^{2}{ }_{\mathrm{w}}$ for low- (DS) and high-pressure (DR) phases of $\mathrm{ZnGa}_{2} \mathrm{Te}_{4}$. Last two columns indicate the EOS type used and the maximum pressure deviation in absolute value $(|\Delta \mathrm{P}|)$ between the EOS and the experiment.

\begin{tabular}{lcccccc}
\hline \hline Phase & $\begin{array}{c}\mathrm{V}_{0} \\
{\left[\AA^{3}\right]}\end{array}$ & $\begin{array}{c}\mathrm{B}_{0} \\
{[\mathrm{GPa}]}\end{array}$ & $\mathrm{B}_{0}{ }^{\prime}$ & $\chi^{2}{ }_{\mathrm{w}}$ & EOS & $\begin{array}{c}|\Delta \mathrm{P}| \\
{[\mathrm{GPa}]}\end{array}$ \\
\hline DS & $414.3(1)$ & $39.8(1)$ & 4 (fixed) & 0.64 & $\mathrm{BM} 2$ & 0.064 \\
DS & $414.3(1)$ & $39.7(5)$ & $4.0(1)$ & 0.68 & $\mathrm{BM} 3$ & 0.063 \\
DR & $192.8(8)$ & $47(1)$ & 4 (fixed) & 1.46 & BM2 & 0.591 \\
DR & $192(1)$ & $50(5)$ & $3.7(4)$ & 1.54 & BM3 & 0.572 \\
\hline \hline
\end{tabular}

DZ phases at ambient pressure it is found that the volume of the DZ phase [399.0(1.2) $\AA^{3}$ ] is between those of the DS [414.14(21) $\left.\AA^{3}\right]$ and DR [385.6(1.6) $\left.\AA^{3}\right]$ phases. In the comparison, the volume for the DR phase at ambient pressure has been obtained from the BM2 EOS, and in the cases of the DR and DZ phases, volume was normalized multiplying by two. The volume of the three phases decreases in the sequence DS $>$ DZ $>$ DR which suggest that the compressibility of the DZ structure should be in between those of the other two phases since the packing efficiency of DZ is in between those of DS and DR.

In order to obtain a direct indication of the compressional behavior of $\mathrm{DS}-\mathrm{ZnGa}_{2} \mathrm{Te}_{4}$ and of the quality of the EOS fit, the $\mathrm{P}-\mathrm{V}$ data are transformed into $F_{E}-f_{E}$ data where $F_{E}\left(f_{E}\right)$ is the normalized stress (finite strain). ${ }^{30}$ The same analysis cannot be done for $\mathrm{DR}-\mathrm{ZnGa}_{2} \mathrm{Te}_{4}$ because its zero-pressure volume cannot be measured since the DR phase is not recovered at ambient pressure. The $F_{E}-f_{E}$ plot for $\mathrm{DS}-\mathrm{ZnGa}_{2} \mathrm{Te}_{4}$ (see Fig. 6) shows that data points lie on near a horizontal line of constant $F_{E}$ corresponding to $\mathrm{B}_{0}{ }^{\prime}=4 .^{30}$ In Fig. 6 it can be seen that a linear fit (dashed blue line) to $F_{E}-f_{E}$ data gives a straight line almost parallel to the case when $\mathrm{B}_{0}{ }^{\prime}=4$ (solid red line). This result indicates that experimental data can be fitted with a BM2, thus

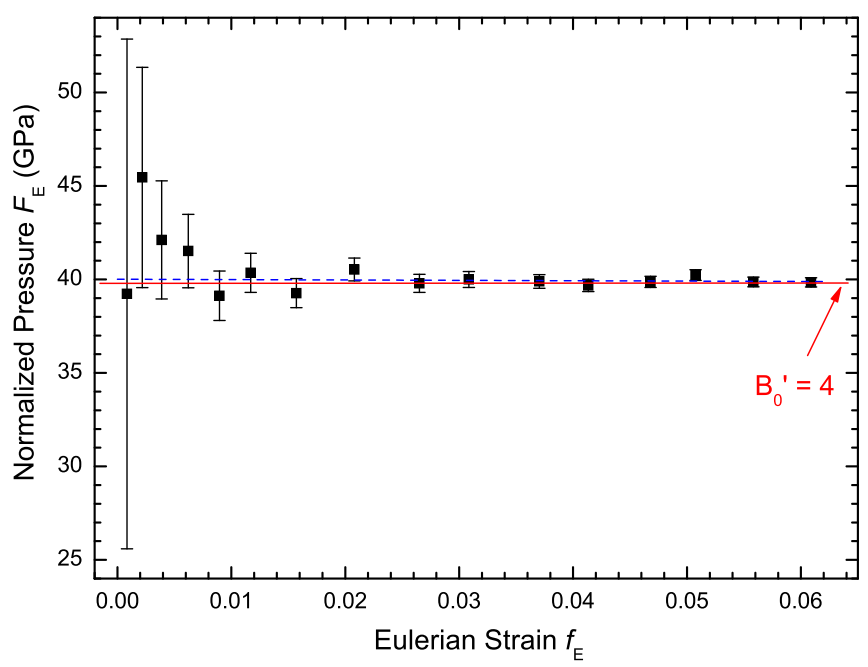

FIG. 6. Volume-pressure data of DS- $\mathrm{ZnGa}_{2} \mathrm{Te}_{4}$ displayed as a plot of the normalized pressure $F_{E}$ against the Eulerian strain $f_{E}$. The blue dashed line is a linear fit to experimental $F_{E}-f_{E}$ data. The solid red line represents the case of a BM2 EOS with $\mathrm{B}_{0}^{\prime}=4$. confirming the validity of the 2 nd order EOS for the measured data. Moreover, a value of $\mathrm{B}_{0}=40.0(3) \mathrm{GPa}$ and $\mathrm{B}_{0}{ }^{\prime}=4.0(1)$ is obtained from the linear fit. In summary, these results confirm that the $\mathrm{BM} 2 \mathrm{EOS}$ is an adequate representation of the P-V data of DS- $\mathrm{ZnGa}_{2} \mathrm{Te}_{4}$ as previously found on the basis of statistical results from the EOS fits.

\section{CONCLUDING REMARKS}

$\mathrm{ZnGa}_{2} \mathrm{Te}_{4}$ has been studied at RT using HP XRD up to 19.8 GPa. An irreversible phase transition has been detected at $\mathrm{P}>12.1 \mathrm{GPa}$ and a third polymorph of $\mathrm{ZnGa}_{2} \mathrm{Te}_{4}$ has been recovered as a metastable phase at ambient pressure. The crystal structure of the three observed polymorphs have been refined and discussed. The low-pressure phase has a defect stannite structure and the high-pressure phase a disordered rocksalt structure. The recovered metastable phase is consistent with a disordered zincblende structure or a subgroup of it. Finally, the axial compressibility of the low-pressure phase, and the RT P-V EOS of the low- and high-pressure phases are reported.

\section{ACKNOWLEDGMENTS}

Research financed by Spain MINECO (MAT201021270-C04-01/04), MALTA Consolider (CSD2007-00045), Generalitat Valenciana (GVA-ACOMP-2013-1012), and Vicerrectorado de Investigación y Desarrollo of UPV (UPV2011-0914 PAID-05-11 and UPV2011-0966 PAID-0611). Part of this work was performed at HPCAT, APS, ANL. HPCAT was supported by CIW, CDAC, UNLV, and LLNL through funding from DOE-NNSA, DOE-BES and NSF. APS was supported by DOE-BES (DEAC02-06CH11357) and UNLV HPSEC by U.S. DOE, NNSA (DE-FC5206NA26274).

${ }^{1}$ S. S. Fouad, G. B. Sakr, I. S. Yahia, and D. M. Abdel Basset, Mater. Res. Bull. 46, 2141 (2011) and references therein.

${ }^{2}$ G. B. Sakr, S. S. Fouad, I. S. Yahia, and D. M. Abdel Basset, J. Mater. Sci. 48, 1134 (2013).

${ }^{3}$ Rashmi and U. Dhawan, Powder Diffr. 17, 41 (2002).

${ }^{4}$ J. Xiao-Shu, Y. Ying-Ce, Y. Shi-Min, M. Shu, N. Zhen-Guo, and L. JiuQing, Chin. Phys. B 19, 107104 (2010).

${ }^{5}$ H. Hahn, G. Frank, W. Klinger, A. D. Störger, and G. Störger, Z. Anorg. Allg. Chem. 279, 241 (1955).

${ }^{6}$ O. Gomis, R. Vilaplana, F. J. Manjón, D. Santamaría-Perez, D. Errandonea, E. Pérez-González, J. López-Solano, P. RodríguezHernández, A. Muñoz, I. M. Tiginyanu, and V. V. Ursaki, J. Appl. Phys. 113, 073510 (2013).

${ }^{7}$ D. Santamaría-Pérez, M. Amboage, F. J. Manjón, D. Errandonea, A. Muñoz, P. Rodríguez-Hernández, A. Mujica, S. Radescu, V. V. Ursaki, and I. M. Tiginyanu, J. Phys. Chem. C 116, 14078 (2012).

${ }^{8}$ S. Meenakshi, V. Vijyakumar, A. Eifler, and H. D. Hochheimer, J. Phys. Chem. Solids 71, 832 (2010).

${ }^{9}$ D. Errandonea, R. S. Kumar, F. J. Manjón, V. V. Ursaki, and I. M. Tiginyanu, J. Appl. Phys. 104, 063524 (2008).

${ }^{10}$ A. Grzechnik, V. V. Ursaki, K. Syassen, I. Loa, I. M. Tiginyanu, and M. Handfland, J. Solid State Chem. 160, 205 (2001).

${ }^{11}$ O. Gomis, R. Vilaplana, F. J. Manjón, D. Santamaría-Pérez, D. Errandonea, E. Pérez-González, J. López-Solano, P. RodríguezHernández, A. Muñoz, I. M. Tiginyanu, and V. V. Ursaki, Mater. Res. Bull. 48, 2128 (2013).

${ }^{12}$ J. Marquina, Ch. Power, P. Grima, M. Morocoima, M. Quintero, B. Couzinet, J. C. Chervin, P. Munsch, and J. González, J. Appl. Phys. 100, 093513 (2006). 
${ }^{13}$ S. Meenakshi, V. Vijyakumar, B. K. Godwal, A. Eifler, I. Orgzall, S. Tkachev, and H. D. Hochheimer, J. Phys. Chem. Solids 67, 1660 (2006).

${ }^{14}$ D. Errandonea, R. S. Kumar, F. J. Manjón, V. V. Ursaki, and E. V. Rusu, Phys. Rev. B 79, 024103 (2009).

${ }^{15}$ O. Gomis, D. Santamaría-Pérez, R. Vilaplana, R. Luna, J. A. Sans, F. J. Manjón, D. Errandonea, E. Pérez-González, P. Rodríguez-Hernández, A. Muñoz, I. M. Tiginyanu, and V. V. Ursaki, J. Alloys Compd. 583, 70 (2014).

${ }^{16}$ O. Gomis, R. Vilaplana, F. J. Manjón, E. Pérez-González, J. LópezSolano, P. Rodríguez-Hernández, A. Muñoz, D. Errandonea, J. RuizFuertes, A. Segura, D. Santamaría-Pérez, I. M. Tiginyanu, and V. V. Ursaki, J. Appl. Phys. 111, 013518 (2012).

${ }^{17}$ I. M. Tiginyanu, N. A. Modovyan, and O. D. Stoika, Sov. Phys. Solid State 43, 527 (1992).

${ }^{18}$ H. K. Mao, J. Xu, and P. M. Bell, J. Geophys. Res. 91, 4673, doi:10.1029/JB091iB05p04673 (1986).

${ }^{19}$ S. Klotz, J. C. Chervin, P. Munsch, and G. Le Marchand, J. Phys. D 42, 075413 (2009).

${ }^{20}$ D. Errandonea, Y. Meng, M. Somayazulu, and D. Häusermann, Physica B 355, 116 (2005)
${ }^{21}$ A. P. Hammersley, S. O. Svensson, M. Hanfland, A. N. Fitch, and D. Häusermann, High Press. Res. 14, 235 (1996).

${ }^{22}$ W. Kraus and G. Nolze, J. Appl. Crystallogr. 29, 301 (1996).

${ }^{23}$ O. Gomis, J. A. Sans, R. Lacomba-Perales, D. Errandonea, Y. Meng, J. C. Chervin, and A. Polian, Phys. Rev. B 86, 054121 (2012).

${ }^{24}$ B. H. Tobby, Powder Diffr. 21, 67 (2006).

${ }^{25}$ R. Vilaplana, O. Gomis, F. J. Manjón, H. M. Ortiz, E. Pérez-González, J. López-Solano, P. Rodríguez-Hernández, A. Muñoz, D. Errandonea, V. V. Ursaki, and I. M. Tiginyanu, J. Phys. Chem. C 117, 15773 (2013).

${ }^{26}$ R. T. Downs and A. K. Singh, J. Phys. Chem. Solids 67, 1995 (2006).

${ }^{27}$ J. Ruiz-Fuertes, A. Friedrich, J. Pellicer-Porres, D. Errandonea, A. Segura, W. Morgenroth, E. Haussühl, C.-Y. Tu, and A. Polian, Chem. Mater. 23, 4220 (2011)

${ }^{28}$ M. D. Frogley, J. L. Sly, and D. J. Dunstan, Phys. Rev. B 58, 12579 (1998).

${ }^{29}$ F. Birch, J. Geophys. Res. 83, 1257, doi:10.1029/JB083iB03p01257 (1978).

${ }^{30}$ R. J. Angel, "Equations of state," in High-Pressure and High-Temperature Crystal Chemistry, edited by R. M. Hazen, R. T. Downs, MSA Reviews in Mineralogy and Geochemistry, vol. 41 (2000), pp. 35-60. 